\title{
Ratio of Transmitral E Wave Velocity to Left Atrial Strain as a Useful Predictor of Total and Cardiovascular Mortality in Hemodialysis Patients
}

\author{
Wei-Chung Tsai ${ }^{1,2,3} \mathbb{D}^{D}$, Wen-Hsien Lee ${ }^{1,3,4}$, Pei-Yu Wu ${ }^{4}$, Jiun-Chi Huang ${ }^{1,3,4} \mathbb{D}$, \\ Ying-Chih Chen ${ }^{4}$, Szu-Chia Chen ${ }^{3,4,5}$ (D), Po-Chao Hsu ${ }^{2,3}$, Chee-Siong Lee ${ }^{2,3}$, \\ Tsung-Hsien Lin ${ }^{2,3}$, Wen-Chol Voon ${ }^{2,3}$ and Ho-Ming Su ${ }^{3,4, *(D)}$ \\ 1 Graduate Institute of Clinical Medicine, College of Medicine, Kaohsiung Medical University, Kaohsiung 807, \\ Taiwan; azygo91@gmail.com (W.-C.T.); cooky-kmu@yahoo.com.tw (W.-H.L.); karajan77@gmail.com (J.-C.H.) \\ 2 Division of Cardiology, Department of Internal Medicine, Kaohsiung Medical University Hospital, \\ Kaohsiung 807, Taiwan; pochao.hsu@gmail.com (P.-C.H.); lcsphk@ms18.hinet.net (C.-S.L.); \\ lth@kmu.edu.tw (T.-H.L.); d750078@kmu.edu.tw (W.-C.V.) \\ 3 Faculty of Medicine, College of Medicine, Kaohsiung Medical University, Kaohsiung 807, Taiwan; \\ scarchenone@yahoo.com.tw \\ 4 Department of Internal Medicine, Kaohsiung Municipal Siaogang Hospital, Kaohsiung 812, Taiwan; \\ wpuw17@gmail.com (P.-Y.W.); 990329kmuh@gmail.com (Y.-C.C.) \\ 5 Research Center for Environmental Medicine, Kaohsiung Medical University, Kaohsiung 807, Taiwan \\ * Correspondence: cobeshm@seed.net.tw; Tel.: +886-7-8036783-3441; Fax: +886-7-8063346
}

Received: 2 December 2019; Accepted: 27 December 2019; Published: 29 December 2019

\begin{abstract}
The ratio of transmitral E-wave velocity (E) to a left ventricular diastolic parameter is reported to be well correlated with left ventricular filling pressure and is useful in the prediction of mortality. Left atrial (LA) strain has been demonstrated to be associated with left ventricular diastolic function. The aim of the study is to examine the ability of E/LA strain in predicting total and cardiovascular mortality in hemodialysis (HD) patients. In 197 routine HD patients, global LA strain during the reservoir phase was estimated by taking the average of longitudinal strain data obtained from the apical four-chamber and two chamber views by two-dimensional speckle tracking echocardiography. Twenty-nine total mortality and 14 cardiovascular mortality were documented during the $2.7 \pm 0.6$-year follow-up. After adjusting age, comorbidities, albumin, E/early diastolic mitral annular velocity (Ea), and LA strain, increased E/LA strain (hazard ratio $(\mathrm{HR})=1.191$, $95 \%$ confidence interval $(\mathrm{CI})=1.072-1.324, p=0.001$ ) was still associated with increased total mortality. After adjusting age, comorbidities, albumin, E/Ea, left ventricular ejection fraction, and LA strain, increased $\mathrm{E} / \mathrm{LA}$ strain $(\mathrm{HR}=1.195,95 \% \mathrm{CI}=1.041-1.372, p=0.011)$ was still associated with increased cardiovascular mortality. In conclusion, E/LA strain is a useful parameter in the prediction of total and cardiovascular mortality in HD patients. Hence, E/LA strain deserves to be calculated in HD patients for better survival prediction.
\end{abstract}

Keywords: total mortality; cardiovascular mortality; left atrial strain; hemodialysis

\section{Introduction}

Cardiovascular disease is the leading cause of mortality in hemodialysis (HD) patients [1]. Calleja et al. found that HD patients had a higher left atrial (LA) volume than normal controls [2]. The left atrium plays an essential role in modulating left ventricular filling, contributing up to a third of cardiac output [3]. Increased LA size has been identified as an important biomarker of left ventricular diastolic dysfunction, cardiovascular disease, and adverse cardiovascular outcomes [4-7]. 
Two-dimensional speckle tracking echocardiography (STE) allows accurate assessment of left ventricular systolic and diastolic function. Recently, STE has proved to be useful in measuring LA mechanics. By measuring peak longitudinal LA strain during the reservoir phase, we can obtain the LA reservoir function. LA reservoir function was reported to be able to predict adverse cardiovascular outcomes both in patients with heart failure with preserved [4] and reduced [8] ejection fraction.

HD patients were found to have a lower LA strain than normal controls [2]. Abid et al. demonstrated that LA strain had a negative correlation with brain natriuretic peptide in HD patients. LA strain has been demonstrated to be associated with left ventricular diastolic function $[9,10]$. By combining the left ventricular diastolic parameter with transmitral $\mathrm{E}$ wave velocity $(\mathrm{E})$, a more accurate estimation of left ventricular filling pressure has been achieved [11-13]. The ratio of $\mathrm{E}$ to early diastolic mitral annular velocity $(\mathrm{Ea})$ was reported to be well related to mean pulmonary capillary wedge pressure and useful in prediction of mortality [12,14]. Hence, we hypothesize the combination index, E/LA strain, also can predict mortality in HD patients. Hence, the present study is designed to examine the ability of LA strain and E/LA strain in the prediction of total and cardiovascular mortality in HD patients.

\section{Materials and Methods}

\subsection{Study Population}

The study was conducted in a regional hospital in southern Taiwan. All regular HD patients in this hospital were included except those who did not accept echocardiographic examination $(n=6)$, those with severe mitral stenosis or mitral regurgitation $(n=3)$, and those with atrial fibrillation $(n=4)$. Finally, 197 patients were included in this study.

\subsection{Ethics Statement}

The study protocol was approved by our institutional review board committee of the Kaohsiung Medical University Hospital (KMUH-IRB). Informed consents were acquired from the patients and our study was conducted according to the principles expressed in the Declaration of Helsinki.

\subsection{Hemodialysis}

All patients received regular HD 3 times per week. Every HD session was done for $3-4 \mathrm{~h}$ using a dialyzer with a blood flow rate of 250 to $300 \mathrm{~mL} / \mathrm{min}$ and a dialysate flow of $500 \mathrm{~mL} / \mathrm{min}$.

\subsection{Echocardiographic Measurements}

The echocardiographic examination was performed using VIVID 7 (General Electric Medical Systems, Horten, Norway) by an experienced echocardiographer who was blind to the clinical data of the patients, according to standardized protocol. Pulsed tissue Doppler imaging was obtained with the sample volume placed at the lateral and septal corners of the mitral annulus from the apical 4-chamber view. Ea was averaged from the septal and lateral ones. The modified Simpson's method was used to evaluate the left ventricular ejection fraction (LVEF). Left ventricular mass was calculated using the Devereux-modified method [15]. The left ventricular mass index (LVMI) was calculated by dividing the left ventricular mass by body surface area. The left atrial volume was calculated using the biplane area-length method [16]. The left atrial volume index (LAVI) was calculated by dividing the left atrial volume by body surface area. The average value of these echocardiographic parameters from 3 consecutive cardiac cycles was used for later analysis.

\subsection{LA Strain Measurement}

LA strain was measured from two-dimensional STE. Detail measurement of LA strain was published in our previous study [17]. Global LA strain during the reservoir phase was calculated by taking the average of longitudinal strain data obtained from the apical four-chamber and two chamber views $[18,19]$. Data acquired from a total of 12 LA segments (annular, mid, and superior segments 
along the septal, lateral, anterior, and inferior LA walls using apical four-chamber and two-chamber images) were averaged to get global longitudinal LA strain at the end of left ventricular ejection (LA reservoir phase). Assessment of LA strain was accepted if at least 4 of the 6 LA segments in each view could be measured clearly. The raw ultrasonic data was recorded and analyzed offline using EchoPAC software (EchoPAC version 08; GE-Vingmed Ultrasound AS GE Medical Systems).

\subsection{Collection of Clinical and Laboratory Data}

Age, sex, current smoking history, and comorbidities were obtained from medical records or interviews with patients. The body mass index (BMI) was calculated as the ratio of weight in kilograms divided by the square of height in meters. Laboratory data were measured from fasting blood samples and obtained within 1 month of enrollment. Diabetes was diagnosed if fasting blood glucose was $\geq 126 \mathrm{mg} / \mathrm{dL}$ or hypoglycemic agents were used to control blood sugar. Stroke was defined as a history of cerebral bleeding or infarction. Coronary artery disease was defined as a history of typical chest pain with a positive stress test, angiographically documented coronary artery disease, old myocardial infarction, or having undergone coronary artery bypass surgery or angioplasty. Heart failure was defined based on the Framingham criteria [20].

\subsection{Definition of Cardiovascular Mortality}

Cardiovascular mortality was determined and judged by two cardiologists with any disagreement resolved by adjudication from a third cardiologist from the hospital course and medical records. Cardiovascular mortality was defined as death caused by ischemia heart disease, cardiogenic shock, heart failure, lethal arrhythmia, unexplained sudden cardiac death, aortic dissection, and cerebrovascular disease. In mortality patients, they were followed until the date of death. The other patients were followed until March 2017.

\subsection{Statistical Analysis}

We used SPSS 22.0 software (SPSS, Chicago, IL, USA) for statistical analysis. Data were presented as mean \pm standard deviation or percentage. Categorical and continuous variables between groups were compared by the chi-square test and independent samples $t$-test, respectively. After we had determined normality using a Kolmogorov-Smirnov test for all continuous variables, appropriate parametric and non-parametric tests were used. We investigated any relationship between two normal distribution variables by Pearson's correlation method and between two non-normal distribution variables by Spearman's correlation method. The significant variables in the univariable linear regression analysis were selected for multivariable linear regression analysis. A Kaplan-Meier survival plot was calculated from baseline to time of mortality events and compared using the log-rank test. Time to mortality events was modeled using the Cox proportional hazards model with a forward selection. All tests were 2-sided and the level of significance was established as $p<0.05$.

\section{Results}

\subsection{Baseline Characteristics in Study Patients}

Table 1 shows the baseline characteristics in our study patients. Among the 197 subjects, the mean age was $61 \pm 12$ years, mean LA strain was $22.1 \pm 7.8 \%$, and mean E/LA strain was $4.2 \pm 2.6 \mathrm{~m} / \mathrm{s}$. 
Table 1. Baseline characteristics in our study patients.

\begin{tabular}{|c|c|}
\hline Characteristics & All Patients $(n=197)$ \\
\hline Age (year) & $61 \pm 12$ \\
\hline Male gender $(\%)$ & 53 \\
\hline Diabetes mellitus (\%) & 47 \\
\hline Current smoking (\%) & 14 \\
\hline CAD $(\%)$ & 10 \\
\hline Stroke $(\%)$ & 10 \\
\hline CHF (\%) & 26 \\
\hline $\mathrm{SBP}(\mathrm{mmHg})$ & $155 \pm 27$ \\
\hline $\mathrm{DBP}(\mathrm{mmHg})$ & $82 \pm 15$ \\
\hline $\operatorname{BMI}\left(\mathrm{kg} / \mathrm{m}^{2}\right)$ & $23.7 \pm 3.8$ \\
\hline Albumin (g/dL) & $3.9 \pm 0.3$ \\
\hline Hemoglobin (g/dL) & $10.5 \pm 1.2$ \\
\hline Total cholesterol (mg/dL) & $178 \pm 40$ \\
\hline Triglyceride (mg/dL) & $167 \pm 123$ \\
\hline \multicolumn{2}{|l|}{ Medications } \\
\hline ACEI and/or ARB use (\%) & 22 \\
\hline$\beta$-blocker use (\%) & 21 \\
\hline CCB use $(\%)$ & 25 \\
\hline \multicolumn{2}{|c|}{ Peripheral vascular parameters } \\
\hline $\mathrm{E}(\mathrm{cm} / \mathrm{s})$ & $83 \pm 30$ \\
\hline $\mathrm{Ea}(\mathrm{cm} / \mathrm{s})$ & $6.7 \pm 2.4$ \\
\hline $\mathrm{E} / \mathrm{Ea}$ & $14.4 \pm 9.5$ \\
\hline LVEF (\%) & $62 \pm 8$ \\
\hline LAVI $\left(\mathrm{mL} / \mathrm{m}^{2}\right)$ & $35 \pm 20$ \\
\hline $\operatorname{LVMI}\left(\mathrm{g} / \mathrm{m}^{2}\right)$ & $136 \pm 42$ \\
\hline LA strain (\%) & $22.1 \pm 7.8$ \\
\hline E/LA strain (m/s) & $4.2 \pm 2.6$ \\
\hline
\end{tabular}

ACEI: angiotensin converting enzyme inhibitor; ARB: angiotensin II receptor blocker; BMI: body mass index; CAD: coronary artery disease; CCB: calcium channel blocker; CHF: chronic heart failure; DBP: diastolic blood pressure; E: transmitral E wave velocity; Ea: early diastolic mitral annular velocity; LA: left atrial; LAVI: left atrial volume index; LVEF: left ventricular ejection fraction; LVMI: left ventricular mass index; SBP: systolic blood pressure.

\subsection{Univariable and Multivariable Correlations of LA strain}

Table 2 shows the univariable and multivariable correlates of LA strain. In the univariable analysis, decreased LA strain was associated with increased age, the presence of diabetes, coronary artery disease, stroke, and chronic heart failure, and decreased triglyceride and Ea. After multivariable analysis, the presence of diabetes and decreased Ea were still the major determinants of decreased LA strain.

\subsection{Univariable and Multivariable Correlations of E/LA strain}

Table 3 shows the univariable and multivariable correlates of E/LA strain. In the univariable analysis, increased E/LA strain was associated with increased age, current smoking, the presence of diabetes, coronary artery disease, and chronic heart failure, using of angiotensin converting enzyme inhibitors/angiotensin II receptor blockers, using of $\beta$ blockers, decreased triglyceride, and increased E/Ea, LAVI, and LVMI. After multivariable analysis, current smoking and increased E/Ea were still the major determinants of increased E/LA strain. 
Table 2. Univariable and multivariable correlates of LA strain in study patients.

\begin{tabular}{lcccc}
\hline \multirow{2}{*}{ Parameter } & \multicolumn{2}{c}{ Univariable Analysis } & \multicolumn{2}{c}{ Multivariable Analysis } \\
\cline { 2 - 5 } & $\boldsymbol{r}$ & $\boldsymbol{p}$ & $\boldsymbol{\beta}$ & $\boldsymbol{p}$ \\
\hline Age (year) & -0.192 & 0.007 & -0.030 & 0.684 \\
Male gender (\%) & -0.066 & 0.360 & & \\
Diabetes mellitus (\%) & -0.327 & $<0.001$ & -0.183 & 0.014 \\
Current smoking (\%) & -0.120 & 0.096 & & \\
CAD (\%) & -0.185 & 0.010 & -0.078 & 0.260 \\
Stroke (\%) & -0.153 & 0.033 & -0.054 & 0.441 \\
CHF (\%) & -0.144 & 0.045 & -0.012 & 0.865 \\
SBP (mmHg) & -0.051 & 0.509 & & \\
DBP (mmHg) & 0.100 & 0.197 & & \\
BMI (kg/m $\left.{ }^{2}\right)$ & -0.134 & 0.062 & & \\
Albumin (g/dL) & 0.033 & 0.650 & & \\
Hemoglobin (g/dL) & -0.043 & 0.553 & & \\
Total cholesterol (mg/dL) & -0.008 & 0.909 & & \\
Triglyceride (mg/dL) & -0.179 & 0.013 & -0.059 & \\
\hline Medications & & & & \\
\hline ACEI and/or ARB use (\%) & -0.133 & 0.064 & & \\
$\beta-$ blocker use (\%) & -0.106 & 0.139 & & \\
CCB use (\%) & -0.091 & 0.209 & & \\
\hline Echocardiographic parameters & & & \\
E (cm/s) & 0.009 & 0.901 & & \\
Ea (cm/s) & 0.378 & $<0.001$ & 0.279 & \\
LVEF (\%) & 0.103 & 0.158 & & \\
LAVI (mL/m ${ }^{2}$ ) & -0.131 & 0.072 & & \\
LVMI (g/m ${ }^{2}$ ) & -0.117 & 0.105 & & \\
\hline & & & & \\
\hline
\end{tabular}

Table 3. Univariable and multivariable correlates of E/LA strain in study patients.

\begin{tabular}{lcccc}
\hline \multirow{2}{*}{ Parameter } & \multicolumn{2}{c}{ Univariable Analysis } & \multicolumn{2}{c}{ Multivariable Analysis } \\
\cline { 2 - 5 } & $\boldsymbol{r}$ & $\boldsymbol{p}$ & $\boldsymbol{\beta}$ & $\boldsymbol{p}$ \\
\hline Age (year) & 0.147 & 0.044 & -0.026 & 0.716 \\
Male gender & 0.108 & 0.142 & & \\
Diabetes mellitus & 0.171 & 0.019 & 0.046 & 0.522 \\
Current smoking & 0.196 & 0.007 & 0.178 & 0.008 \\
CAD & 0.222 & 0.002 & 0.113 & 0.121 \\
Stroke & 0.017 & 0.820 & & \\
CHF & 0.190 & 0.009 & 0.040 & 0.591 \\
SBP (mmHg) & 0.004 & 0.963 & & \\
DBP (mmHg) & -0.103 & 0.190 & & \\
BMI (kg/m ${ }^{2}$ ) & 0.067 & 0.366 & & \\
Albumin (g/dL) & 0.026 & 0.721 & & \\
Hemoglobin (g/dL) & -0.022 & 0.768 & & \\
Total cholesterol (mg/dL) & 0.014 & 0.852 & & \\
Triglyceride (mg/dL) & 0.155 & 0.035 & -0.024 & \\
\hline Medications & & & & 0.727 \\
\hline ACEI and/or ARB use & 0.206 & 0.005 & 0.019 & \\
-blocker use & 0.157 & 0.032 & -0.025 & 0.759 \\
CCB use (\%) & 0.123 & 0.095 & & \\
\hline Echocardiographic parameters & & & \\
\hline E/Ea & 0.596 & $<0.001$ & 0.399 & $<0.001$ \\
LVEF (\%) & 0.047 & 0.525 & & \\
LAVI (mL/m ${ }^{2}$ ) & 0.304 & $<0.001$ & 0.133 & \\
LVMI (g/m ${ }^{2}$ ) & 0.183 & 0.012 & 0.007 & 0.922 \\
\hline & & & & \\
\hline
\end{tabular}




\subsection{Kaplan-Meier Analyses of Total and Cardiovascular Mortality-free Survival in Our Patients}

The follow-up period to mortality was $2.7 \pm 0.6$ years. Twenty-nine total mortalities and 14 cardiovascular mortalities were recognized during the follow-up period. The causes of mortality included chronic heart failure with acute exacerbation $(n=7)$, acute myocardial infarction $(n=3)$, cerebrovascular disease $(n=3)$, lethal arrhythmia $(n=1)$, malignancy $(n=5)$, infectious disease $(n=9)$, and gastrointestinal bleeding $(n=1)$.

The best cut-off value of E/LA strain in the prediction of mortality has not been established. To find the appropriate cut-off value of E/LA strain as a predictor of cardiovascular mortality, we created some models using different cut-off values of E/LA strain. Using the chi-square value to choose the model with the best performance, the model using E/LA strain $>4.56 \mathrm{~m} / \mathrm{s}$ had the best performance in predicting cardiovascular mortality. There were 61 patients with E/LA strains of $>4.56 \mathrm{~m} / \mathrm{s}$. Figure 1 illustrates the Kaplan-Meier curves for total mortality-free survival (Figure 1A) and cardiovascular mortality-free survival (Figure 1B) in study patients subdivided according to E/LA strain $>4.56 \mathrm{~m} / \mathrm{s}$ or not $(\log$-rank $p \leq 0.012)$. Patients with $\mathrm{E} / \mathrm{LA}$ strain $>4.56 \mathrm{~m} / \mathrm{s}$ had higher total and cardiovascular mortality rates.
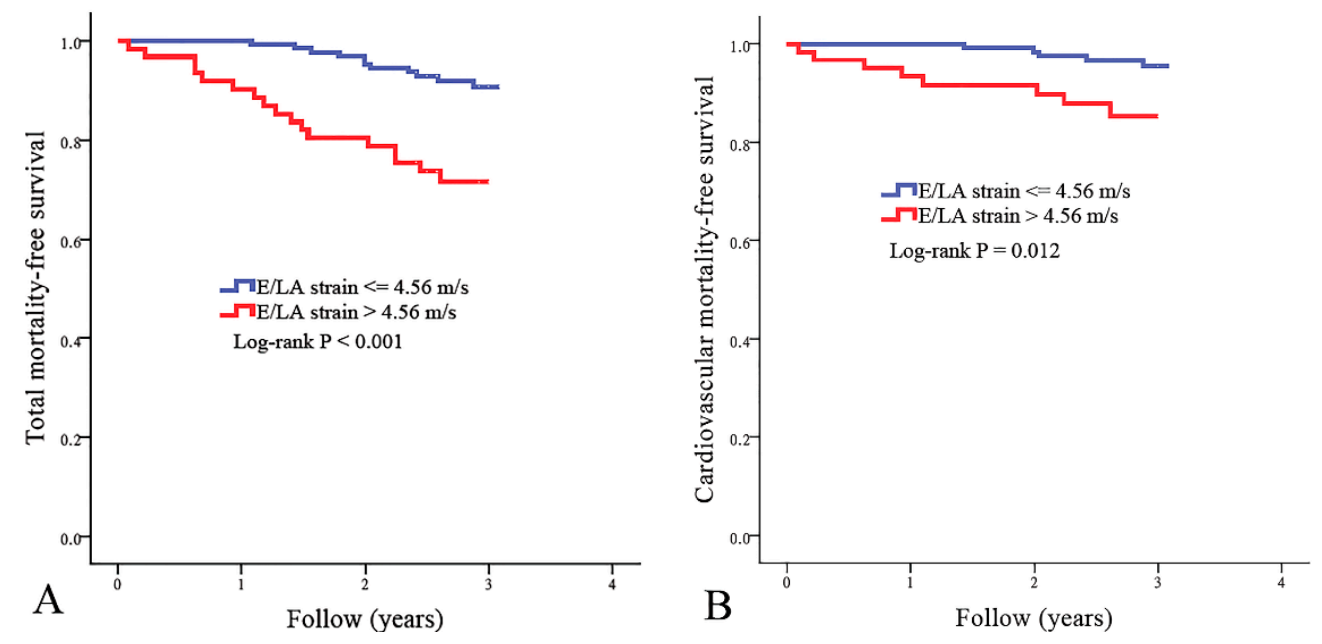

Figure 1. Kaplan-Meier curves for total mortality-free survival ((A), log-rank $p<0.001)$ and cardiovascular mortality-free survival $((\mathbf{B}), \log$-rank $p=0.012)$ in all study patients subdivided according to the ratio of transmitral E wave velocity (E) to left atrial (LA) strain $>4.56 \mathrm{~m} / \mathrm{s}$ or not.

\subsection{Major Predictors of Total and Cardiovascular Mortality in Study Patients}

Table 4 shows the predictors of total mortality using the Cox proportional hazards model. In the univariable analysis, increased total mortality was associated with increased age, E/Ea, and E/LA strain, the presence of diabetes, coronary artery disease, and chronic heart failure, and decreased albumin and LA strain. After multivariable analysis, increased age and E/LA strain, the presence of coronary artery disease, and decreased albumin were still the major predictors of increased total mortality.

Table 5 shows the predictors of cardiovascular mortality using the Cox proportional hazards model. In the univariable analysis, increased cardiovascular mortality was associated with increased age, E/Ea, and E/LA strain, the presence of diabetes and coronary artery disease, and decreased albumin, LVEF, and LA strain. After multivariable analysis, increased age, the presence of coronary artery disease, and increased E/LA strain were still the major predictors of increased cardiovascular mortality. 
Table 4. Predictors of total mortality using the Cox proportional hazards model.

\begin{tabular}{lcccc}
\hline \multirow{2}{*}{ Parameter } & \multicolumn{2}{c}{ Univariable Analysis } & \multicolumn{2}{c}{ Multivariable Analysis (Forward) } \\
\cline { 2 - 5 } & HR (95\% CI) & $p$ & HR (95\% CI) & $p$ \\
\hline Age (year) & $1.070(1.034-1.107)$ & $<0.001$ & $1.086(1.036-1.138)$ & 0.001 \\
Male gender & $0.947(0.457-1.962)$ & 0.883 & & \\
Diabetes mellitus & $3.275(1.449-7.339)$ & 0.003 & & \\
Current smoking & $1.030(0.358-2.959)$ & 0.957 & & \\
CAD & $4.392(1.939-9.950)$ & $<0.001$ & $4.965(1.963-12.557)$ & \\
CHF & $2.153(1.028-4.509)$ & 0.037 & & \\
SBP (mmHg) & $1.009(0.994-1.025)$ & 0.250 & & \\
DBP (mmHg) & $1.004(0.977-1.032)$ & 0.775 & & \\
BMI (kg/m $\left.{ }^{2}\right)$ & $0.909(0.814-1.015)$ & 0.094 & & \\
Albumin (g/dL) & $0.197(0.088-0.441)$ & $<0.001$ & $0.276(0.100-0.764)$ & \\
Hemoglobin (g/dL) & $0.859(0.634-1.164)$ & 0.330 & & \\
\hline Medications & & & & \\
\hline ACEI and/or ARB use & $1.482(0.655-3.351)$ & 0.342 & & \\
3-blocker use & $1.263(0.539-2.958)$ & 0.591 & & \\
CCB use & $1.895(0.895-4.013)$ & 0.089 & & \\
\hline Echocardiographic parameters & & & \\
\hline E/Ea & $1.028(1.005-1.053)$ & 0.015 & & \\
LVEF (\%) & $0.970(0.932-1.009)$ & 0.128 & & \\
LAVI (mL/m $\left.{ }^{2}\right)$ & $1.005(0.995-1.016)$ & 0.318 & & \\
LA strain $\%)$ & $0.929(0.881-0.979)$ & 0.006 & & \\
E/LA strain (m/s) & $1.167(1.072-1.171)$ & $<0.001$ & $1.191(1.072-1.324)$ & \\
\hline
\end{tabular}

$\mathrm{HR}$, hazard ratio; $\mathrm{CI}$, confidence interval.

Table 5. Predictors of cardiovascular mortality using the Cox proportional hazards model.

\begin{tabular}{|c|c|c|c|c|}
\hline \multirow{2}{*}{ Parameter } & \multicolumn{2}{|c|}{ Univariable Analysis } & \multicolumn{2}{|c|}{ Multivariable Analysis (Forward) } \\
\hline & HR (95\% CI) & $p$ & HR (95\% CI) & $p$ \\
\hline Age (year) & $1.059(1.007-1.112)$ & 0.025 & $1.096(1.028-1.170)$ & 0.005 \\
\hline Male gender & $1.598(0.535-4.768)$ & 0.397 & & \\
\hline Diabetes mellitus & $4.620(1.287-16.585)$ & 0.010 & & \\
\hline Current smoking & $1.746(0.487-6.260)$ & 0.386 & & \\
\hline CAD & $6.421(2.136-19.300)$ & $<0.001$ & $6.403(1.848-22.182)$ & 0.003 \\
\hline $\mathrm{CHF}$ & $2.285(0.793-6.590)$ & 0.116 & & \\
\hline SBP (mmHg) & $1.017(0.995-1.039)$ & 0.125 & & \\
\hline DBP (mmHg) & $1.024(0.989-1.061)$ & 0.182 & & \\
\hline BMI $\left(\mathrm{kg} / \mathrm{m}^{2}\right)$ & $0.935(0.802-1.091)$ & 0.397 & & \\
\hline Albumin (g/dL) & $0.221(0.069-0.714)$ & 0.013 & & \\
\hline Hemoglobin (g/dL) & $0.859(0.634-1.164)$ & 0.330 & & \\
\hline \multicolumn{5}{|l|}{ Medications } \\
\hline ACEI and/or ARB use & $2.199(0.735-6.586)$ & 0.148 & & \\
\hline$\beta$-blocker use & $1.605(0.503-5.125)$ & 0.420 & & \\
\hline CCB use & $1.723(0.577-5.142)$ & 0.324 & & \\
\hline \multicolumn{5}{|c|}{ Echocardiographic parameters } \\
\hline $\mathrm{E} / \mathrm{Ea}$ & $1.031(1.000-1.065)$ & 0.049 & & \\
\hline LVEF (\%) & $0.941(0.896-0.989)$ & 0.015 & & \\
\hline LAVI $\left(\mathrm{mL} / \mathrm{m}^{2}\right)$ & $1.008(0.996-1.020)$ & 0.176 & & \\
\hline LA strain $(\%)$ & $0.919(0.852-0.993)$ & 0.031 & & \\
\hline E/LA strain (m/s) & $1.202(1.074-1.345)$ & 0.001 & 1.195 (1.041-1.372) & 0.011 \\
\hline
\end{tabular}




\section{Discussion}

This study aimed to evaluate LA strain and E/LA strain in the prediction of total and cardiovascular mortality in HD patients. We found the combination index, E/LA strain, was significantly associated with total and cardiovascular mortality after multivariable adjustment in HD patients.

Nagy et al. found reduced LA strain but not left ventricular systolic and diastolic parameters were associated with heart failure symptoms and outcome in patients with heart failure with preserved ejection fraction [21]. Malagoli et al. reported LA strain could predict cardiovascular events in patients with chronic heart failure with reduced ejection fraction [22]. In contrast, Modin et al. demonstrated that LA strain is a univariable predictor of cardiovascular morbidity and mortality in the general population. However, LA strain did not remain an independent predictor of outcome after adjustment for clinical parameters [23]. Hence, in patients without heart failure, LA strain might not be a helpful outcome predictor. In the present study, although LA strain was significantly associated with total and cardiovascular mortality in the univariable analysis, the association became insignificant after multivariable adjustment. In HD patients, LA strain might not be a useful parameter in mortality prediction.

Diastolic dysfunction may increase left ventricular filling pressure and is the important mechanism responsible for the pathophysiology of heart failure. By combining the left ventricular diastolic parameters with transmitral E-wave velocity, a more precise estimate of left ventricular filling pressure is achieved. The ratio of transmitral $\mathrm{E}$ wave velocity to left ventricular diastolic parameter, such as E/Ea and E/left ventricular early diastolic strain rate, had a significant correlation with left ventricular filling pressure $[12,24]$ and could usefully predict mortality in various populations, including in patients with heart failure with reduced ejection fraction [25], in the general population [25], and in patients with acute myocardial infarction [26,27]. Many previous studies have shown that LA strain has a significant correlation with left ventricular diastolic function [28-30]. In the present study, we also found LA strain had a significant correlation with $\mathrm{Ea}$, an established left ventricular diastolic parameter, so the combination index, E/LA strain, should have a significant correlation with left ventricular filling pressure and a potential to predict mortality. In fact, our results showed E/LA strain was highly correlated with E/Ea, a good parameter of left ventricular filling pressure [12] and after adjusting many important clinical and echocardiographic parameters, including E/Ea, LVMI, and LA strain, E/LA strain was still a useful parameter in the prediction of total and cardiovascular mortality in our HD patients. Hence, assessment of the combination index, E/LA strain, is helpful in identifying the high-risk group of increased total and cardiovascular mortality in HD patients.

\section{Study limitation}

There were several limitations to this study. First, the study generality was restricted because we only included study patients from one dialysis clinic in a regional hospital in southern Taiwan. Second, two-dimensional STE can generate LA strain and strain rate curves among different LA cycle phases. We only calculated longitudinal LA strain during the reservoir phase. However, LA strain during the reservoir phase has been considered to be the most well-studied one in predicting mortality among the various LA strain measurements [21,22]. Third, because of the abnormally large left atrium in patients with severe mitral stenosis or mitral regurgitation and the lack of effective atrial contraction in patients with atrial fibrillation, we did not include such patients. Finally, due to the large number of variables in our analysis with only 29 total mortality events and 14 cardiovascular mortality events, the possibility of chance findings and the restricted power should be considered.

\section{Conclusions}

In HD patients, the combination index, E/LA strain, was significantly associated with total and cardiovascular mortality after adjusting several important clinical and echocardiographic parameters. Hence, E/LA strain deserves to be measured in HD patients for better survival prediction. 
Author Contributions: Conceptualization, W.-C.T., W.-H.L. and H.-M.S.; Data curation, W.-C.T., P.-C.H., J.-C.H. and T.-H.L.; Formal analysis, W.-C.T., W.-H.L. and H.-M.S.; Funding acquisition, W.C.T., Y.-C.C. and H.-M.S.; Investigation, W.-C.T., J.-C.H., Y.-C.C., S.-C.C. and P.-Y.W.; Methodology, J.-C.H., Y.-C.C., S.-C.C., P.-Y.W. and H.-M.S.; Project administration, W.-H.L., S.-C.C. and W.-C.V.; Resources, P.-Y.W., T.-H.L. and C.-S.L.; Software, C.-S.L. and H.-M.S.; Supervision, S.-C.C., C.-S.L., W.-C.V. and H.-M.S.; Validation, P.-Y.W., T.-H.L., and W.-C.V.; Visualization, T.-H.L. and H.-M.S.; Writing—original draft, W.-C.T. and W.-H.L.; Writing-review \& editing, S.-C.C., W.-C.V. and H.-M.S. All authors have read and agreed to the published version of the manuscript.

Funding: Our study is supported by the grant from Kaohsiung Municipal Siaogang Hospital (kmhk-108-002), Kaohsiung Medical University, Kaohsiung, Taiwan

Conflicts of Interest: The authors declare no other conflict of interest.

\section{References}

1. Mallick, N.P.; Jones, E.; Selwood, N. The european (european dialysis and transplantation association-european renal association) registry. Am. J. Kidney Dis. 1995, 25, 176-187. [CrossRef]

2. Calleja, A.M.; Rakowski, H.; Williams, L.K.; Jamorski, M.; Chan, C.T.; Carasso, S. Left atrial and ventricular systolic and diastolic myocardial mechanics in patients with end-stage renal disease. Echocardiogr. 2016, 33, 1495-1503. [CrossRef] [PubMed]

3. Matsuda, Y.; Toma, Y.; Ogawa, H.; Matsuzaki, M.; Katayama, K.; Fujii, T.; Yoshino, F.; Moritani, K.; Kumada, T.; Kusukawa, R. Importance of left atrial function in patients with myocardial infarction. Circulation 1983, 67, 566-571. [CrossRef] [PubMed]

4. Santos, A.B.; Roca, G.Q.; Claggett, B.; Sweitzer, N.K.; Shah, S.J.; Anand, I.S.; Fang, J.C.; Zile, M.R.; Pitt, B.; Solomon, S.D.; et al. Prognostic relevance of left atrial dysfunction in heart failure with preserved ejection fraction. Circ. Heart Fail. 2016, 9, e002763. [CrossRef]

5. Lee, J.S.; Shim, C.Y.; Wi, J.; Joung, B.; Ha, J.W.; Lee, M.H.; Pak, H.N. Left ventricular diastolic function is closely associated with mechanical function of the left atrium in patients with paroxysmal atrial fibrillation. Circ. J. 2013, 77, 697-704. [CrossRef]

6. Santos, A.B.; Kraigher-Krainer, E.; Gupta, D.K.; Claggett, B.; Zile, M.R.; Pieske, B.; Voors, A.A.; Lefkowitz, M.; Bransford, T.; Shi, V.; et al. Impaired left atrial function in heart failure with preserved ejection fraction. Eur. J. Heart Fail. 2014, 16, 1096-1103. [CrossRef]

7. Gerdts, E.; Wachtell, K.; Omvik, P.; Otterstad, J.E.; Oikarinen, L.; Boman, K.; Dahlof, B.; Devereux, R.B. Left atrial size and risk of major cardiovascular events during antihypertensive treatment: Losartan intervention for endpoint reduction in hypertension trial. Hypertension 2007, 49, 311-316. [CrossRef]

8. Carluccio, E.; Biagioli, P.; Mengoni, A.; Francesca Cerasa, M.; Lauciello, R.; Zuchi, C.; Bardelli, G.; Alunni, G.; Coiro, S.; Gronda, E.G.; et al. Left atrial reservoir function and outcome in heart failure with reduced ejection fraction. Circ. Cardiovasc. Imaging 2018, 11, e007696. [CrossRef]

9. Wakami, K.; Ohte, N.; Asada, K.; Fukuta, H.; Goto, T.; Mukai, S.; Narita, H.; Kimura, G. Correlation between left ventricular end-diastolic pressure and peak left atrial wall strain during left ventricular systole. J. Am. Soc. Echocardiogr. 2009, 22, 847-851. [CrossRef]

10. Cameli, M.; Mandoli, G.E.; Loiacono, F.; Dini, F.L.; Henein, M.; Mondillo, S. Left atrial strain: A new parameter for assessment of left ventricular filling pressure. Heart Fail. Rev. 2016, 21, 65-76. [CrossRef]

11. Gonzalez-Vilchez, F.; Ares, M.; Ayuela, J.; Alonso, L. Combined use of pulsed and color m-mode doppler echocardiography for the estimation of pulmonary capillary wedge pressure: An empirical approach based on an analytical relation. J. Am. Coll. Cardiol. 1999, 34, 515-523. [CrossRef]

12. Nagueh, S.F.; Middleton, K.J.; Kopelen, H.A.; Zoghbi, W.A.; Quinones, M.A. Doppler tissue imaging: A noninvasive technique for evaluation of left ventricular relaxation and estimation of filling pressures. J. Am. Coll. Cardiol. 1997, 30, 1527-1533. [CrossRef]

13. Mitter, S.S.; Shah, S.J.; Thomas, J.D. A test in context: E/a and e/e' to assess diastolic dysfunction and lv filling pressure. J. Am. Coll. Cardiol. 2017, 69, 1451-1464. [CrossRef] 
14. Kim, M.K.; Kim, B.; Lee, J.Y.; Kim, J.S.; Han, B.G.; Choi, S.O.; Yang, J.W. Tissue doppler-derived e/e' ratio as a parameter for assessing diastolic heart failure and as a predictor of mortality in patients with chronic kidney disease. Korean J. Intern. Med. 2013, 28, 35-44. [CrossRef] [PubMed]

15. Devereux, R.B.; Alonso, D.R.; Lutas, E.M.; Gottlieb, G.J.; Campo, E.; Sachs, I.; Reichek, N. Echocardiographic assessment of left ventricular hypertrophy: Comparison to necropsy findings. Am. J. Cardiol. 1986, 57, 450-458. [CrossRef]

16. Lang, R.M.; Bierig, M.; Devereux, R.B.; Flachskampf, F.A.; Foster, E.; Pellikka, P.A.; Picard, M.H.; Roman, M.J.; Seward, J.; Shanewise, J.S.; et al. Recommendations for chamber quantification: A report from the american society of echocardiography's guidelines and standards committee and the chamber quantification writing group, developed in conjunction with the european association of echocardiography, a branch of the european society of cardiology. J. Am. Soc. Echocardiogr. 2005, 18, 1440-1463.

17. Hsu, P.C.; Lee, W.H.; Chu, C.Y.; Lee, H.H.; Lee, C.S.; Yen, H.W.; Lin, T.H.; Voon, W.C.; Lai, W.T.; Sheu, S.H.; et al. Prognostic role of left atrial strain and its combination index with transmitral e-wave velocity in patients with atrial fibrillation. Sci. Rep. 2016, 6, 17318. [CrossRef]

18. Pathan, F.; D'Elia, N.; Nolan, M.T.; Marwick, T.H.; Negishi, K. Normal ranges of left atrial strain by speckle-tracking echocardiography: A systematic review and meta-analysis. J. Am. Soc. Echocardiogr. 2017, 30, 59-70. [CrossRef]

19. Meel, R.; Khandheria, B.K.; Peters, F.; Libhaber, E.; Nel, S.; Essop, M.R. Left atrial volume and strain parameters using echocardiography in a black population. Eur. Heart J. Cardiovasc. Imaging 2017, 18, 350-355. [CrossRef]

20. Ho, K.K.; Anderson, K.M.; Kannel, W.B.; Grossman, W.; Levy, D. Survival after the onset of congestive heart failure in framingham heart study subjects. Circulation 1993, 88, 107-115. [CrossRef]

21. Nagy, A.I.; Hage, C.; Merkely, B.; Donal, E.; Daubert, J.C.; Linde, C.; Lund, L.H.; Manouras, A. Left atrial rather than left ventricular impaired mechanics are associated with the pro-fibrotic st2 marker and outcomes in heart failure with preserved ejection fraction. J. Intern. Med. 2018, 283, 380-391. [CrossRef] [PubMed]

22. Malagoli, A.; Rossi, L.; Bursi, F.; Zanni, A.; Sticozzi, C.; Piepoli, M.F.; Villani, G.Q. Left atrial function predicts cardiovascular events in patients with chronic heart failure with reduced ejection fraction. J. Am. Soc. Echocardiogr. 2019, 32, 248-256. [CrossRef] [PubMed]

23. Modin, D.; Biering-Sorensen, S.R.; Mogelvang, R.; Alhakak, A.S.; Jensen, J.S.; Biering-Sorensen, T. Prognostic value of left atrial strain in predicting cardiovascular morbidity and mortality in the general population. Eur. Heart J. Cardiovasc. Imaging 2019, 20, 804-815. [CrossRef]

24. Lassen, M.C.H.; Sengelov, M.; Qasim, A.; Jorgensen, P.G.; Bruun, N.E.; Olsen, F.J.; Fritz-Hansen, T.; Gislason, G.; Biering-Sorensen, T. Ratio of transmitral early filling velocity to early diastolic strain rate predicts all-cause mortality in heart failure with reduced ejection fraction. J. Card. Fail. 2019, 25, 877-885. [CrossRef] [PubMed]

25. Lassen, M.C.H.; Biering-Sorensen, S.R.; Olsen, F.J.; Skaarup, K.G.; Tolstrup, K.; Qasim, A.N.; Mogelvang, R.; Jensen, J.S.; Biering-Sorensen, T. Ratio of transmitral early filling velocity to early diastolic strain rate predicts long-term risk of cardiovascular morbidity and mortality in the general population. Eur. Heart J. 2019, 40, 518-525. [CrossRef] [PubMed]

26. Kruszewski, K.; Scott, A.E.; Barclay, J.L.; Small, G.R.; Croal, B.L.; Moller, J.E.; Oh, J.K.; Hillis, G.S. Noninvasive assessment of left ventricular filling pressure after acute myocardial infarction: A prospective study of the relative prognostic utility of clinical assessment, echocardiography, and b-type natriuretic peptide. Am. Heart J. 2010, 159, 47-54. [CrossRef]

27. Ersboll, M.; Andersen, M.J.; Valeur, N.; Mogensen, U.M.; Fakhri, Y.; Thune, J.J.; Moller, J.E.; Hassager, C.; Sogaard, P.; Kober, L. Early diastolic strain rate in relation to systolic and diastolic function and prognosis in acute myocardial infarction: A two-dimensional speckle-tracking study. Eur. Heart J. 2014, 35, 648-656. [CrossRef]

28. Fernandes, R.M.; Le Bihan, D.; Vilela, A.A.; Barretto, R.B.M.; Santos, E.S.; Assef, J.E.; Pedra, S.R.F.; Sousa, A.; Timerman, A. Association between left atrial strain and left ventricular diastolic function in patients with acute coronary syndrome. J. Echocardiogr. 2019, 17, 138-146. [CrossRef] 
29. Jarasunas, J.; Aidietis, A.; Aidietiene, S. Left atrial strain-An early marker of left ventricular diastolic dysfunction in patients with hypertension and paroxysmal atrial fibrillation. Cardiovasc. Ultrasound 2018, 16, 29. [CrossRef]

30. Dogan, C.; Ozdemir, N.; Hatipoglu, S.; Bakal, R.B.; Omaygenc, M.O.; Dindar, B.; Candan, O.; Emiroglu, M.Y.; Kaymaz, C. Relation of left atrial peak systolic strain with left ventricular diastolic dysfunction and brain natriuretic peptide level in patients presenting with st-elevation myocardial infarction. Cardiovasc. Ultrasound 2013, 11, 24. [CrossRef]

(C) 2019 by the authors. Licensee MDPI, Basel, Switzerland. This article is an open access article distributed under the terms and conditions of the Creative Commons Attribution (CC BY) license (http://creativecommons.org/licenses/by/4.0/). 